\title{
Perceptual organization and consciousness
}

D. Samuel Schwarzkopf \& Geraint Rees

Wellcome Dept of Imaging Neuroscience, Institute of Neurology, University College London, UK

To appear in:

Oxford Handbook of Perceptual Organization

Oxford University Press

Edited by Johan Wagemans

\section{Introduction}

All of our lives revolve around our conscious experience of the world we inhabit. In spite of that, the question of why we have consciousness in the first place and how much it influences our perception and action remains largely unanswered. Is consciousness just an epiphenomenon, a genetic quirk that arose in the course of evolution as a consequence of other processes in the human brain, or does it have a teleological purpose? For vision, this interpretation depends not only on the object or feature that is the current focus of attention, but also on the perceptual context in which it is embedded. Yet surprisingly little is currently understood about how perceptual organisation affects our consciousness, whether conscious awareness of sensory stimuli is a prerequisite for interpreting them as coherent objects and scenes, and the underlying neural processes in the human brain.

This chapter will review the state of research on how consciousness is entwined with the perceptual organisation of the sensory input. The first section, Access to consciousness, describes the categorical nature of how our conscious perception is typically viewed and how this can be used to make inferences about the neural correlates of consciousness. The following section, Unconscious perceptual organization, will go into more depth on the interaction between awareness of a stimulus and the brain's interpretation of it. This also includes a discussion of studies trying to address the question whether there is any information that requires conscious awareness of the stimulus to be processed. The final section, Phenomenological contents of consciousness, describes research going beyond the purely categorical aspects of our awareness but instead concentrating on the mechanisms determining a person's percept of the environment.

\section{Access to consciousness}

We are all familiar with ways in which our awareness and our perception interact. At any point in time, our sense organs are bombarded by an overwhelming amount of input; however, we are usually not aware of this information overload. Rather, we usually feel that we are only really conscious of a particular part or aspect of the environment. Moreover, some aspects of our sensorium are usually or almost always outside our awareness (James 1890). For example, we are generally unaware of our heartbeat or of the workings of our internal organs even though there are afferent nerves continuously transmitting signals to our brain. Only when something requires our attention, for example when we are hungry or sick, we usually feel anything about our bodies and even then it is merely a vague feeling not a thorough awareness of all our affected bodily functions. Thus the focus of awareness constantly fluctuates, partly under our own volition and partly for reasons that are mostly outside our control.

Studies investigating the neural events that determine whether a sensation reaches consciousness and what kind of perceptual processing occurs unconsciously can take several forms. One obvious approach is to 
manipulate directly whether the observer is aware of the sensory stimulus. In the visual domain this is typically done through masking procedures of which there are numerous variations. It is possible to mask a stimulus from being consciously perceived by presenting a masking stimulus either directly after or before the onset of the stimulus, methods known as masking, respectively. Among such methods, meta-contrast masking (Breitmeyer and Ogmen 2000) employs masking stimuli with contours of opposite contrast polarity to the stimulus of interest being presented subsequent to that stimulus. This method can render even bright stimuli invisible to the observer. An extension to this method employs a mask presented for a longer period before the stimulus of interest. Repeating this several times results in a "standing wave of invisibility" that can render a stimulus invisible for prolonged periods (Macknik and Livingstone 1998). This methodology can show that information about stimulus orientation is present in primary visual cortex (V1) even when the orientation does not reach awareness (Haynes and Rees 2005), consistent with behavioural experiments showing that grating stimuli rendered invisible through various forms of masking can produce contextual interactions or adaptation effects on contrast or orientation perception (Clifford and Harris 2005; Falconbridge et al. 2010; Motoyoshi and Hayakawa 2010).

While such methods can be very effective in removing a stimulus from conscious access and typically allow excellent experimental control over awareness, they share the caveat that they are based on substantial perturbations of the stimulus and therefore it becomes difficult to distinguish the effect of changes in the stimulus parameters from changes in consciousness. It is unsurprising that a stimulus presented in close temporal proximity to the stimulus will interfere with the neuronal response to that stimulus (Macknik and Livingstone 1998). Nevertheless, this approach can provide important insights about what distinguishes conscious and unconscious processing as long as this stimulus confound is taken into account. In essence, if a stimulus can exert unconscious effects when rendered invisible through masking (or any other stimulus manipulation), this is sufficient evidence that it is processed even in the absence of awareness. However, when no unconscious effects are observed the interpretation is more complicated. The only direct conclusion that can be made in this situation is that the processing of a stimulus is disrupted by this stimulus manipulation. Further inference on the role of conscious awareness can only be made through convergent evidence combining other masking procedures or different manipulations of awareness.

Another popular approach to studying unconscious processing is therefore to directly exploit the fluctuating focus of awareness. To do this one can use multistable perception. Ambiguous images, like those shown in Figure 1, can be interpreted in more than one way but only one interpretation is ever experienced at a time. The dynamics and behavioural studies of ambiguous images are discussed in detail in the chapter by Alais and Blake (this volume). For example, the Necker cube (Figure 1A) can be perceived such that the upper corner is either facing forward or facing backward. Sometimes a third state is reported in which the impression of depth is lost entirely - a two-dimensional collection of parallelograms. Critically, however, it is impossible to see all of these interpretations simultaneously.

Under ideal situations, comparing the variable percept evoked by such ambiguous images dissociates the contents of awareness (which alternate) from physical stimulation (which remains unchanging). Naturally, this is based on the assumption that peripheral processes in the individual perceiving these stimuli are constant between the different perceptual experiences. This may not be the case in all situations. For example, subtle eye movements may change the retinal projection of the Necker cube and favour one interpretation of the two-dimensional image over another (Einhäuser et al. 2004). In this context it is also worth noting that eye movements do not correspond with perceived depth of a stimulus but reflect lowlevel attributes of the image (Wismeijer et al. 2008, 2010). For ambiguous structure-from-motion stimuli that lead to perception of a three dimensional shape spinning either clockwise or anti-clockwise, the percept may depend on whether attention is directed to the dots drifting to the left or to the right. Moreover, for many ambiguous stimuli one of the interpretations is more dominant. Thus, provided such 
peripheral factors are controlled for adequately this approach permits a stronger inference to be made about the neural correlates of consciousness than manipulating the stimulus directly. However, by using multistable stimuli one loses direct experimental control over the observer's conscious perceptual experience.

One particular form of bistable perception occurs when two different stimuli are presented to separate paired sensory organs, so that the brain receives conflicting sensory inputs. This has been studied most extensively with binocular rivalry, when each eye is presented with a different image. Rather than seeing an incoherent mixture or blend of the two images, conscious perception typically alternates between each monocular percept just as with other types of ambiguous stimuli. A third piecemeal percept, where the perceived image is a mosaic of images seen by the left and right eyes can also occur. During the switches between alternate interpretations, perception does not flip instantaneously from one state to another but changes rapidly from an initiating location across the visual field, akin to a wave travelling across the image. Psychophysical studies of binocular rivalry and such perceptual waves also receive much greater attention in the chapter by Alais and Blake (this volume).

Of course, the eyes are not the only sensory organs that come as a pair. Therefore, it is unsurprising that there are equivalents of binocular rivalry for other senses. In binaural rivalry, the two ears hear different sequences of tones. The resulting percept alternates between the specific sensations rather than evoking a cacophony of mismatching sounds (van Ee et al. 2009; Brancucci and Tommasi 2011). Even more surprising, in binaral rivalry two different odours are presented separately to each of the nostrils and again the perceived smell switches back and forth between the two (Zhou and Chen 2009). Unlike binocular rivalry, which occurs naturally under normal viewing conditions outside Panum's fusional area, binaural and binaral rivalry are sensory conditions that must be artificially created in a laboratory. In the normal environment of an organism it is not probable that each of the nostrils would receive conflicting smells or that completely different sounds would reach each of the ears without any crossover between the two. On the other hand, in natural vision the image projected onto the two retinas are generally quite distinct and there are frequent occurrences where two completely different images are seen at least by parts of each retina: for example, the region blocked by the nose. Moreover, outside Panum's fusional area binocular fusion does not occur. Fusing the two retinal images in a meaningful way is the basis of stereovision and thus important for judging depth and distance. Thus, binocular rivalry is an extreme situation that reveals a mechanism associated with normal visual processing away from fixation. Binaural and binaral rivalry on the other hand seem to be a purer demonstration of the processes underlying the wavering focus of awareness. It is therefore of note that in spite of this, the three forms of bi-sensory rivalry are phenomenologically very similar.

Perhaps the simplest form of bistable perception occurs when two stimuli are superimposed or mixed. In the visual domain this is sometimes referred to as monocular rivalry, that is, when the same picture contains two different images. Again, the focus of perception can alternate between the two individual images. Even though this effect may not have the same potency as binocular rivalry or other ambiguous images, it underlines that all of the sensory input cannot be processed simultaneously with equal processing resources. We can focus on one component image but only perceive the other one as a distracting background blur or vice versa (O'Craven et al. 1999); alternatively, we may force vision to perceive both at the same time but this only results in a messy, broken up percept. 

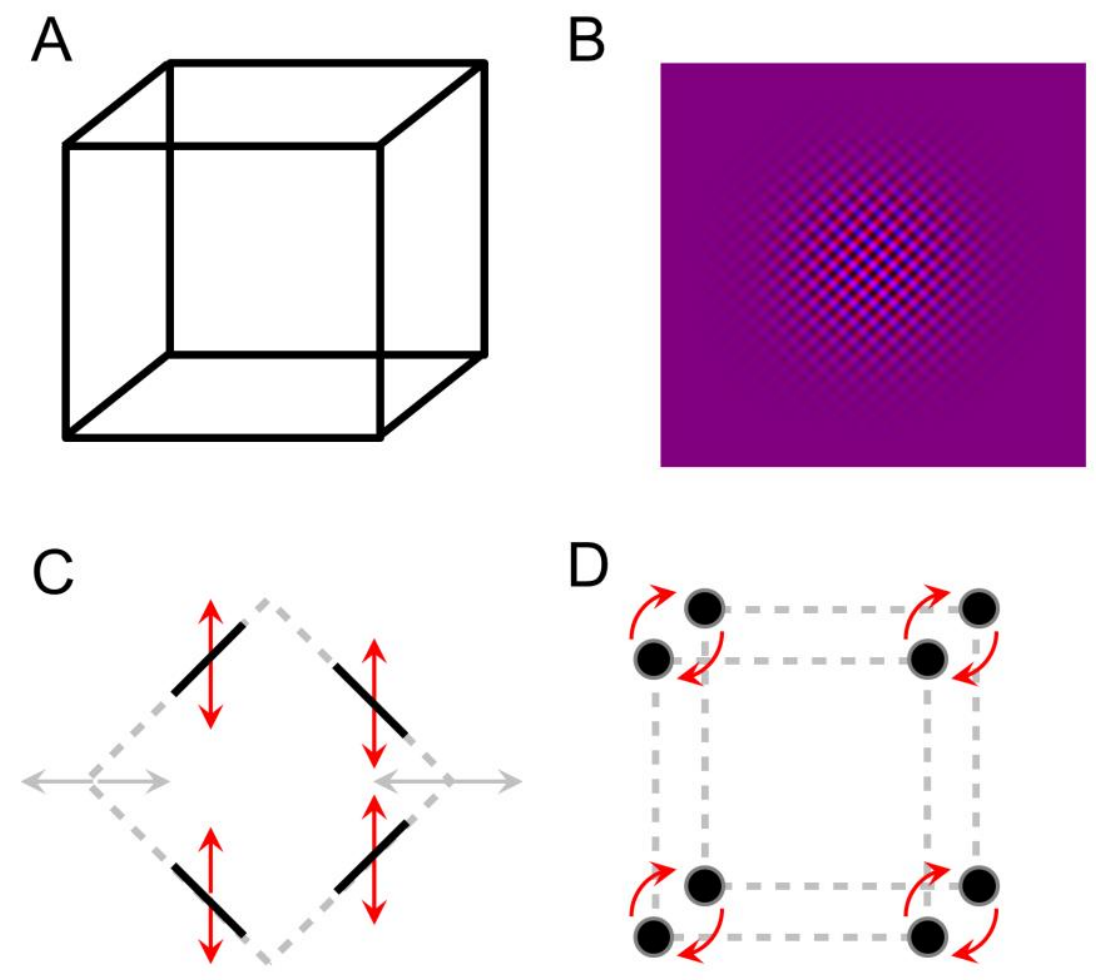

Figure 1. Examples of ambiguous stimuli showing both traditional examples $(A, B)$ and stimuli that become multistable because of changes in how the visual system interprets low-level information $(C, D)$. A. The Necker Cube for which perception alternates between which face is interpreted as being in front. B. Binocular rivalry. When viewing this stimulus with red-blue anaglyph glasses perception alternates between the two oblique grating patches (see the chapter by Alais and Blake for an in-depth discussion and more examples). C. Even though only the black bars are visible and physically moving up and down (denoted by red arrows), perception can interpret this stimulus also as a black diamond shape (implied by the dashed grey lines) viewed behind white, vertical occluding bars. (Please refer to http://www.pnas.org/content/suppl/2002/10/26/192579399.DC1/5793Movie2Legend.html for a moving demonstration. D. Each of the four pairs of discs constantly circles around a hinge point (denoted by red arrows). We can interpret this locally as four pairs of discs, but perception can also be dominated by a global interpretation in which there are two groups of four dots arranged in the squares implied by the dashed lines. Please refer to http://psy2.ucsd.edu/ sanstis/Stuart_Anstis/Local_and_Global.html for a moving demonstration and a discussion of the parameters determining whether the local or global interpretation predominates.

It should also be noted that the fact that perception can be multistable at all has implications for our understanding of the perceptual apparatus. The reason that we are not conscious of all possible interpretations of an ambiguous stimulus could be related to a limit in the capacity with which the brain can perceptually organise and interpret the overwhelming sensory input. However, if this is true, this must mean that some information can only be processed with awareness of the stimulus. Conversely, the fact that our percept does not simply stabilise into one of the possibilities is inconsistent with any account that the brain merely interprets the sensorium using the most probable prior expectation. Instead perhaps the continuous fluctuation in perception reflects the brain's way to search for the appropriate solution when faced with strongly ambiguous input. Reconciling theories of prediction with rivalrous perception remains an important topic for future research. 
What neural processes underlie the perceptual switches and periods of perceptual dominance in multistable perception? The advent of modern neuroimaging techniques like positron emission tomography (PET), functional magnetic resonance imaging (fMRI), electroencephalography (EEG), and magnetoencephalography (MEG) have made it possible to measure neural activity throughout the human brain whilst measuring behavioural reports of the observer's perceptual state in real time. Such experiments show that regions of superior parietal and prefrontal cortex, which are also associated with attentional deployment, are active during the transitions from one perceptual state to another (Lumer et al. 1998). Moreover, the structure of such regions is related to the frequency of perceptual switches. Specifically, individual differences in the grey matter volume in right superior parietal cortex correlate with the switch rate for a structure-from-motion stimulus (Kanai et al. 2010). Causally manipulating neural activity in these regions using transcranial magnetic stimulation (TMS) with continuous theta-burst stimulation decreases switch rate (Kanai et al. 2010) showing that these areas play a causal role in generating perceptual switches. Moreover, applying TMS to a slightly more anterior part of parietal cortex has the opposite effect on switch rates in binocular rivalry (Carmel et al. 2010; Zaretskaya et al. 2010). Taken together this suggest a sophisticated model in with parietal (and perhaps prefrontal) cortices play a complex causal role in generating top-down signals that ultimately resolve perceptual competition in ventral visual cortex (Kanai et al. 2011).

The link between brain structure and the switch rate in these forms of perceptual rivalry also hints at the possibility that these processes are deeply rooted in human physiology. While grey matter volume can change over the life span and there is some short-term experience-dependent plasticity associated with learning motor tasks (Draganski et al. 2004), there is a strong heritable component to switch rate in multistable perception (Miller et al. 2010; Shannon et al. 2011). Moreover, switch rate correlates with the occurrence and severity of bipolar disorder (Pettigrew and Miller 1998; Miller et al. 2003; Krug et al. 2008; Nagamine et al. 2009). This obviously does not imply that binocular rivalry, and bistable perception in general, is causal to psychiatric or neurological conditions but it suggests that rivalry shares mechanisms affected in these conditions. Recent studies investigated the balance of excitatory and inhibitory signalling in visual cortex motivated by the assumption that this balance relates to the dynamics of perceptual rivalry (van Loon et al. 2013), which could be altered in certain conditions (Aznar Casanova et al. 2013; Said et al. 2013).

Naturally, the focus of awareness does not exist in isolation from wider perceptual processing. While there is a strong stochastic element to how and when perceptual transitions occur during multistable perception, the timing of such transitions is also strongly influenced by the stimuli used and other factors, such as what stimuli had been presented previously or attentional deployment. So it is possible to some degree to control perceptual alternations through selectively attending to one particular interpretation (Ooi and He 1999; Hugrass and Crewther 2012), although binocular rivalry may be less susceptible to voluntary control than other forms of multistability (Meng and Tong 2004). Moreover, when viewing of a binocular rivalry stimulus is interrupted by a blank epoch, the first percept reported when the rivalrous stimulus returns is frequently the same as the one last perceived before the blank epoch (Leopold et al. 2002). Even more fundamentally, basic image statistics can influence bistable perception. During binocular rivalry, sharp edges with high contrasts and sudden movement usually result in perceptual dominance, while homogeneous regions of an image tend to be suppressed. Thus rivalrous images that contain a large degree of heterogeneity in one eye but homogenous regions in the other tend to be dominated by the heterogeneous image. The sudden appearance of one monocular image can substantially bias the percept to being dominated by that image, a process known as flash suppression (Wolfe 1984), perhaps because sudden appearance of a stimulus is particularly salient (Cole et al. 2004). 
This phenomenon can be exploited to sustain perceptual dominance of one eye for prolonged periods. One eye views a dynamic stream of constantly changing patterns of high-contrast geometric shapes (e.g. a Mondrian-like pattern) while the other views a low contrast stimulus. Under the right circumstances such continuous flash suppression (CFS) results in complete dominance of perception for extended periods of time by the dynamic stimulus, thus suppressing the other monocular stimulus from awareness (Tsuchiya and Koch 2005). It is however critical to keep in mind that this suppression may differentially affect the lowlevel stimulus components, such as the stimulus spatial frequency (Yang and Blake 2012) and the phase alignment of stimulus and mask (Maehara et al. 2009). CFS has been used to study unconscious stimulus processing in numerous studies and enjoys increasing popularity due to the ease of its use. In one variant of these experiments, the contrast of the suppressed image is gradually increased and the critical parameter to be measured is the "time to emergence" when the suppressed stimulus breaks through the masking stimulus in the other eye and reaches awareness. Comparing this parameter for different stimulus conditions can reveal differences in the unconscious processing of the images (Jiang et al. 2007). However, it is always important to keep in mind the time it takes a stimulus to break interocular suppression may be determined not necessarily by a stimulus parameter of interest but could also involve other, low-level features of the suppressed image. Further, it is possible that a faster time to emergence does not actually reflect unconscious processing but rather the speed (or other dynamics) with which the stimulus breaks through suppression once it has passed the threshold to conscious perception.

At an even more basic level, image statistics vie for perceptual dominance. When one eye views white noise images, while the other views noise images filtered to fall within the $1 / f$ spectrum typically observed in natural scenes (Field 1987; Simoncelli and Olshausen 2001), the latter dominate perception for a significantly longer periods than the white noise images (Baker and Graf 2009). This may suggest that the visual system selectively responds by bringing stimuli whose image statistics conform with the natural world to the focus of awareness. However, the same may not apply to higher order image statistics, such as the collinearity or co-circularity of orientated segments in the image. While some studies show that collinear gratings in a binocular rivalry stimulus tend to perceptually transition as a group (Alais and Blake 1999), there have also been reports that when a noisy field of grating patches of random orientations is paired with a field of varying levels of co-circularity in the other eye, it is the incoherent, random pattern that dominates perception (Hunt et al. 2012), even though the natural environment contains a high degree of such co-circular regularities (Geisler et al. 2001; Geisler 2008). The reason for that may be that the two monocular images in that study were not perfectly overlapping, so that individual patches were not in directly rivalry with one another. Particular relevant to the question how the visual system organises stimulus elements into coherent objects it is interesting that interocular suppression spreads along contours and around angles and even across gaps in a contour provided that it is interpreted as arising from occlusion (Maruya and Blake 2009). It is evident that the same processes that are involved with organising our perception into a coherent representation of the environment have complex interactions with awareness.

Bistability of the contents of awareness can also be experienced with regard to how the brain interprets information as a coherent whole. A stimulus like that shown in Figure $1 \mathrm{C}$ can be perceived in different states, reflecting the way individual stimulus elements are regarded as being independent or part of a larger object (Murray et al. 2002; Fang, Kersten, et al. 2008). In the local state the two lines are perceived as drifting up or down, i.e. the veridical interpretation. However, in the global state the observer instead reports the lines as the sides of a square that is moving left and right behind several occluding rectangles. Which particular interpretation currently dominates perception also influences the aftereffects from using these stimuli as adaptors (He et al. 2012). A similar stimulus is shown in Figure 1D. There are four groups of stimuli, each comprising two discs circling around a central hinge point. Under the local interpretation, each of these groups are perceived as independent moving objects (perhaps akin to binary star systems). However, in the global state discs from distant locations are grouped into larger entities, resulting in the percept of two squares rotating around one another. Neuroimaging experiments show that in the global 
state, neural responses in early visual cortex to such stimuli are reduced relative to the local interpretation (Zaretskaya et al. 2013). Such a response pattern is a hallmark of coherent perceptual organisation, possibly indicative of predictive coding by which areas higher up in the processing hierarchy send feedback signals to early visual cortex that cancel out the neural activity that is "explained away" by coherent objects (Rao and Ballard 1999; Murray et al. 2002; Joo et al. 2012). However, such an interpretation is complicated by the fact that while responses in early visual cortex are reduced, this reduction is general to the whole region, rather than specifically to the location responding to the stimulus (de-Wit et al. 2012). Moreover, the neural representation of the stimulus is enhanced (Kok et al. 2012), which could be related to the fact that there is reduced variability in stimulus features (Dumoulin and Hess 2006) and thus reduced lateral inhibition (which would appear as metabolic activity in neuroimaging measurements) between adjacent neuronal populations with different tuning properties (Kinoshita et al. 2009). While such lower level explanations cannot entirely account for findings supporting the predictive coding hypothesis in the context of ambiguous stimuli, the underlying neural mechanisms are probably more complicated than the predictive coding account proposes.

The beauty of these particular stimulus examples lies in the fact that like all bistable images, the stimuli themselves are physically constant and only perceptual organisation alternates. However, one problem with these particular forms of bistable perceptual organisation is that our interpretation is typically fairly biased towards one state. For instance, in the latter example the percept becomes more predominantly local as the speed of rotation is increased and more critically it tends to become more global with prolonged exposure (Anstis and Kim 2011). This is also why it is necessary to continuously adapt stimulus parameters to ensure relatively equal dominance of each state (Zaretskaya et al. 2013), something that is typically less problematic for more classical ambiguous stimuli like binocular rivalry or structure-from-motion displays that continuously switch between perceptual states. Nevertheless, as these and other studies illustrate, stimuli like these can be used successfully to reveal how grouping processes influence the contents of awareness.

One way to reveal neural correlates of consciousness and to understand what information is processed in the absence of awareness is to rely entirely on whether a stimulus gains access to conscious report or not. Multistability is not the only means of doing this. For example, there have been demonstrations of priming effects exerted by stimuli that remained undetected in change blindness paradigms (Silverman and Mack 2006; Yeh and Yang 2009). Interestingly, while previous neuroimaging and transcranial magnetic stimulus (TMS) experiments implicate right parietal and dorsolateral prefrontal cortex in signalling for the presence of a change of the stimulus (Beck et al. 2001, 2006; Turatto et al. 2004), there is also evidence to suggest that the memory trace of a stimulus can be boosted by applying TMS to visual cortical areas encoding the stimulus (Schwarzkopf et al. 2010).

Research on the neural correlates of consciousness (Rees et al. 2002) has also implicated that recurrent connectivity between brain regions in the sensory hierarchy is critically important for conscious perception of a stimulus. The visibility of a visual stimulus under metacontrast masking correlates with effective connectivity between early visual areas and fusiform cortex, which seems to relate to activity in the region immediately surrounding the retinotopic representation of the stimulus in early visual cortex (Haynes et al. 2005). Further, it has been proposed that feedback from higher regions into earlier areas is critical for conscious perception (Roelfsema et al. 1998; Lamme and Roelfsema 2000; Lamme 2006) although others have argued that at least for visual masking paradigms consciousness varies due to disruptions in feedforward processing (Tse et al. 2005; Dehaene et al. 2006; Macknik and Martinez-Conde 2007). 


\section{Unconscious perceptual organisation}

Bistability is not the only phenomenon that can illustrate interactions between perceptual organisation and awareness. The contents of awareness are modulated by many factors that can depend on our perceptual organisation. One such phenomenon is motion-induced blindness (Bonneh et al. 2001). Here a few small target stimuli, yellow dots, are placed inside a dark background containing a rotating grid of blue dots. After viewing this stimulus (and maintaining stable eye fixation) one of the yellow dots will vanish, completely blotted out by the surrounding dynamic background (see http://www.michaelbach.de/ot/motmib/index.html for a demonstration). The neural processes underlying this effect remain unclear. Activity in retinotopic regions corresponding to the target location is modulated upon its disappearance. The pattern of modulation is complex with decreased activity in ventral region V4 accompanied by somewhat counterintuitive increases in dorsal and early visual cortex (Donner et al. 2008; Schölvinck and Rees 2010). Interestingly, the probability of disappearance is also enhanced when attention is directed to the target (Schölvinck and Rees 2009).

A similar phenomenon is the artificial scotoma that occurs when we view a small plain target on a background of high-contrast, dynamic noise. After prolonged viewing the target is perceptually filled in by the background and vanishes from awareness (Ramachandran and Gregory 1991). This process is not dissimilar to the filling in that occurs in the blind spot corresponding to the optic disc in the eye or with scotomas resulting from damage to the retina. Such filling in and perceptual extrapolation mechanisms can be so effective that the individual themselves is not even aware that there is anything abnormal with their vision. Neuroimaging experiments show that the disappearance of the target stimulus is accompanied by a reduction in target-related neural responses in early visual cortices (Weil et al. 2007, 2008). These examples demonstrate how our perceptual apparatus continuously works to interpret the sensory input and extrapolates across gaps in the sensory representation to generate a more coherent representation of the world in our mind's eye.

The plethora of perceptual phenomena related to consciousness, both in terms of awareness of an object's presence or the fluctuating focus of our perceptual interpretation of the sensory input, show that awareness and perceptual organisation are closely intertwined. But to what extent does awareness influence perception? Because the neural nature of consciousness remains very poorly understood, the role it plays in our interpretation of the environment is also difficult to establish. Are there any perceptual functions that require conscious awareness of the stimulus? Alternatively, could consciousness be simply a product of the mind but irrelevant for how the brain analyses sensory information?

There have been numerous demonstrations of how unconscious stimuli can have complex and powerful effects on behaviour. Images of emotional faces rendered invisible through masking, can influence behavioural performance (Yang et al. 2007; Faivre et al. 2012; Almeida et al. 2013) and produce brain activation in neuroimaging experiments linked to emotional processing, like enhanced amygdala responses to fearful faces (Williams et al. 2004; de Gelder et al. 2005). This suggests that the neural mechanisms required for detecting emotional expressions operate even when we are not aware of the stimulus. Similar findings have been made for social information in faces. For example, the time for a face to emergence from continuous flash suppression (i.e. the time it takes for a low contrast face stimulus to break through the dichoptic mask) is influenced by its dominance or trustworthiness (Stewart et al. 2012). It has been argued that the information about emotional valence, in particular fear responses, is confined to low spatial frequencies and bypasses the high-resolution image analysis in early visual cortex entirely (Vuilleumier et al. 2003; Winston et al. 2003) through a subcortical pathway. This would suggest that while perceptual analysis necessary for such primal emotional responses is independent of awareness, conscious processing may nevertheless be required for detailed perceptual organisation. 
However, even more complex information is processed in the absence of awareness. For example, semantic information can be processed without awareness and break through binocular suppression (Costello et al. 2009), although it is unclear how much semantic information can be processed whilst undergoing dichoptic suppression (Zimba and Blake 1983). Organising local image features like lines and angles into letters, and subsequently letters into words, must require fairly sophisticated processing. At least to some extent this process must be preserved in the absence of conscious awareness. Whether or not an invisible stimulus exerts an influence on perception probably also depends on what aspect of perception is measured: while a high-order visual stimulus, like a spiral, may not produce adaptation (unlike simpler stimuli, like a grating) when masked from awareness, a complex, naturalistic image may still capture attentional resources (Lin and He 2009). Further, as discussed earlier, one important aspect to consider is also that the means by which a stimulus is rendered invisible may influence whether a stimulus can have subliminal effects (Faivre et al. 2012; Yang and Blake 2012). A briefly presented stimulus followed by a mask may be available to complete perceptual processing even though it is unavailable to conscious report. On the other hand, presenting the same stimulus under conditions under binocular rivalry may eliminate its neural representation in higher brain regions where the information about the stimulus eye of origin is lost.

In light of this problem, it is even more interesting that even the processing of complex natural images appears to proceed under continuous flash suppression that renders the images invisible. One study measured the time to emergence to visual scenes that were either congruent with the natural world or contained some form of inconsistency, such as an archer using a tennis racket instead of an arrow or basketball players using a watermelon instead of a ball (Mudrik et al. 2011). Intriguingly, incongruent scenes broke through perceptual suppression faster than congruent scenes. This may suggest that even the complex integration of objects in their semantic context can occur in the absence of awareness. Even if we assume that this effect may in some way be influenced by low level image properties (an explanation which is somewhat unlikely due to the diverse range of natural stimuli used in that study) and bypasses detailed visual analysis through different pathways, it must require some complex processes to identify the out-ofplace features. This finding is in some way contrary to the aforementioned reports of a bias for more "natural" stimuli to dominate in binocular rivalry (Baker and Graf 2009). However, as discussed in the previous section it is also important to note that the measure used by this study, time to emergence from CFS, may not truly reflect the processing that occurs under suppression but the detection of incongruent scenes at the moment of transition between suppression and visibility, which in turn results in them reaching perceptual dominance with a faster speed.

In contrast to this finding, the neural representation of complex visual stimuli may not be the same in the absence of awareness as during conscious viewing. For example, one study used multivariate pattern decoding techniques to decode distributed activations measured with high-resolution functional MRI in higher ventral visual cortex to distinguish processing associated with viewing of face or house images (Sterzer et al. 2008). While it was possible to decode which of the two stimulus classes was being processed, regardless of whether or not they were rendered invisible by continuous flash suppression, the results suggested that the nature of the pattern information under awareness and invisibility was different. This is notably different from the situation in early visual cortex where the neural representation of invisible orientated gratings is similar to visible stimuli (Haynes and Rees 2005). The overall visual response in higher visual brain regions to stimuli rendered invisible through binocular fusion (when two complimentary images are presented to each eye and perceived merely as a uniform blank) can be very similar, albeit weaker, as that to visible stimuli (Moutoussis and Zeki 2002; Schurger et al. 2010). This suggests that there may be fundamental differences in terms of how information about the visual stimulus is encoded during unconscious processing. 
It has been argued that one neural correlate of awareness is the reliability of the visual response to the stimulus (Schurger et al. 2010). Using functional MRI and multivariate decoding analysis these authors showed that the pattern of activation produced by invisible stimuli is indeed more variable compared to that for visible stimuli. However, it seems curious to regard this as a neural correlate of consciousness: by definition variability must be determined over the course of multiple or prolonged measurements. Consciousness, on the other hand, can vary from one moment to the next. While it is certainly possible that one property granting neural representations access to consciousness may be its temporal stability, the response patterns in functional MRI are measured on a trial by trial basis with each trial comprising slow haemodynamic measurements over several seconds. It seems unlikely that response variability between such trials can explain the absence (or presence) of awareness across all trials because awareness of a stimulus operates at much faster time scales. More importantly, because this study employed a stimulus manipulation (binocular fusion) to render the stimulus invisible it is a demonstration of the earlier discussion of masking methods: it is impossible to rule out that the reduced reliability of fMRI responses is correlated to consciousness or merely a result of differences in the stimulus. Only a design that compares conscious and unconscious trials with identical stimulation can conclusively arbitrate between those possibilities.

Nevertheless, the finding is interesting because it suggests that without awareness a stabilising influence on the neural representation may be lost. This is also supported by psychophysical experiments showing that without awareness, behavioural sensitivity to orientation is broader consistent with greater variability (Ling and Blake 2009). In that study, awareness was manipulated by using binocular rivalry with flash suppression and comparing identical stimulus conditions in the presence and absence of awareness, rather than directly manipulating the stimulus to render it invisible. This provides stronger evidence that the differences indeed relate to consciousness rather than physical differences in visual input.

Another interesting aspect of Schurger and colleagues' finding was that the brain regions where the most diagnostic information about the visual images was encoded differed between visible and invisible stimuli (Schurger et al. 2010). While the former selectively activated well-replicated areas in ventral cortex known to respond preferentially to images of faces and houses, respectively, invisible stimuli on the other hand were decoded by more posterior regions in intermediate fusiform cortex presumably corresponding to areas V4 and the VO complex (Wandell et al. 2007). While these regions are already sensitive to relatively complex visual information, they are not as selective for object identity. It is therefore possible that in the absence of awareness visual information is encoded in a more incoherent form, relying on more primitive features rather than abstract classes. At least some perceptual organisation, transforming geometric primitives into coherent and meaningful objects, may thus require consciousness.

To test this notion, in behavioural experiments we measured priming effects produced by simple visual shapes that were either visible or rendered invisible by fast counter-phase flicker, a method that seems to allow for at least low-level processing of visual information to occur (Falconbridge et al. 2010). Shapes comprised sparse fragments and could either be defined by the position or the orientation of the elements (Schwarzkopf and Rees 2010). We observed that priming effects from invisible stimuli on the discrimination of shapes of the opposite feature only occurred when the primes were defined by orientation. Moreover, this effect disappeared when the discrimination targets were rescaled. This indicates that without awareness, oriented elements are not integrated into an abstract representation of a shape but that some more local processes involved in spatial integration, possibly confined to early retinotopic cortex, are nonetheless functioning. Consciousness, it seems, is after all required for some more abstract analysis of the visual environment. 
This notion was also supported by an experiment in which we tested whether Kanizsa triangles are formed when the inducers producing this type of illusory contour are rendered invisible by continuous flash suppression, but a central region containing the illusory contour produced by the stimulus configuration was available to conscious perception (Harris et al. 2011). Participants were required to discriminate the orientation of the illusory contour. Without awareness, performance was consistently at chance levels indicating that participants could not perceive the illusory contour. This contrasts with a control experiment where we showed that simultaneous brightness contrast (Figure $2 \mathrm{~A}$ ), the contextual modulation of perceived brightness when a stimulus is presented against a dark or light background, is preserved even when the background is suppressed from awareness. This null finding for perception of illusory contours when the inducers are suppressed from awareness cannot be explained by lack of statistical power, because each participant performed a large number of trials and performance was extremely consistent across the group. However, as previously discussed with any of these studies in which awareness is manipulated by a change in the stimulus, it is possible that the dichoptic masking procedure, rather than consciousness per se, interfered with the formation of the illusory contours. Others have shown that when masking illusory contours are not perceived when the inducers are suppressed during binocular rivalry (Sobel and Blake 2003). There is evidence that illusory contours are mediated by binocular neurons (Liu et al. 1994; Gillam and Nakayama 1999; Häkkinen and Nyman 2001) that may have been affected by dichoptic masking. One argument speaking against that is that Kanizsa triangles enhance the speed with which a stimulus breaks through binocular suppression (Wang et al. 2012), although this is inconsistent with the absence of any effect on dominance periods during binocular rivalry (Sobel and Blake 2003), although it remains unclear in how far the time to emergence from binocular suppression reflects unconscious processing per se.

It is also likely that inferring illusory contours operates through a multi-stage process where first the local stimulus features are segmented and grouped into objects which then produces the illusory percept possibly mediated by hierarchically earlier stages of visual processing through feedback (Kogo et al. 2010); see also the chapter by Kogo and van Ee, this volume). This is consistent with the finding that stimuli that mimic the salience of Kanizsa figures but that do not produce the percept of illusory contours produce similar neural responses in lateral occipital cortex, a region presumed to be involved in extracting surfaces and objects (Stanley and Rubin 2003). It also agrees with recent findings that the perception of Kanizsa stimuli depends not only on processing in early visual cortex but also on feedback from higher lateral occipital cortex (Wokke et al. 2013). The arrangement of the inducers may attract attention to the Kanizsa stimulus without producing an actual percept of illusory contours. This is not an unlikely explanation because there is considerable evidence that, while related, attention is a process distinct from awareness (Kentridge et al. 1999; Lamme 2003; Koch and Tsuchiya 2007; Bahrami et al. 2008a, 2008b; Zhaoping 2008). Further, the spread of attentional responses in V1 is determined by Gestalt principles (Wannig et al. 2011). The extent to which processing of illusory contours occurs without awareness thus still remains a question to be resolved by future research. However, our results already point towards the fact that illusory contours are formed at least at a higher-level stage of processing beyond where signals from the two eyes are still separate.

Interestingly in this context, there have been findings from stroke patients with parietal extinction (where a stimulus on the side contralateral to a parietal lesion remains undetected if a simultaneous ipsilateral stimulus is presented. Grouping of stimuli that form Kanizsa figures can alleviate the effects of extinction (Mattingley et al. 1997; Conci et al. 2009) suggesting that these processes can are not dependent on awareness of the stimulus. However, again in this situation it is unclear which comes first, the production of illusory contours or the segmentation of stimuli into surfaces. This line of research is discussed in greater detail in the chapter by Gillebert and Humphreys (this volume). 


\section{Phenomenological contents of consciousness}

Thus far we have considered consciousness in terms of the focus and contents of the mind's eye. However, the concept of the contents of awareness is broader than merely whether we are aware of a stimulus or not. Perception of objects is strongly modulated by interactions with their neighbours and the context in which they appear. Contextual illusions like simultaneous brightness contrast and other examples shown in Figure 3 reveal processes by which the visual system shapes perception of objects rather than representing a physically accurate reality. These processes serve a teleological purpose because they reflect the way the brain interprets the small, inherently two-dimensional images falling on the retinae in the eyes as originating in a large, three-dimensional world. Perceiving the same luminance object with different brightness depending on whether it is brightly lit or in the shade, or two objects with identical retinal size as bigger or smaller depending on how far away we believe it to be, are mechanisms for interpreting sensory input in a meaningful way. Our perception may be "fooled" by illusions, because they are tailored around the way perceptual processing works; however, in the real world this processing typically helps us understand that an object close to us is not oppressively large even though it covers most of the visual field. The visual system is not designed for making photometric measurements or precise estimations of visual angle. Its purpose is to help the observer understand and interpret the environment and forming a representation about their place in the world.

Through these modulations of our sensory input illusions alter the contents of consciousness. Rather than simply determining what we perceive at all, consciousness also reflects how we perceive the world around us. Because they disentangle the physical reality of the stimulus from our subjective experience of it, they are also excellent tools for research into how consciousness interacts with perceptual organisation and of the underlying neuronal mechanisms.

Typically, these illusions rely on the fact that physically identical stimuli can appear notably different depending on either the surround they appear in or on other global interpretations. We already mentioned simultaneous brightness contrast (Figure 2A) where the brightness of a stimulus is influenced by the brightness of the surround. Similar effects are seen in the tilt illusion where the orientation of a central grating appears to be tilted away from that of a surrounding annulus, contrast-suppression (Figure 2B) where the contrast of a central stimulus surrounded by a high-contrast annulus appears to be reduced, and the Ebbinghaus illusion (Figure $2 \mathrm{C}$ ) where a stimulus appears larger or smaller depending on the size of and the distance to stimuli surrounding it (Roberts et al. 2005). Other illusions, like the Ponzo and Mueller-Lyer illusions (Figure 2D,E) and variants thereof, may affect the neural processes underpinning interpretation of three-dimensional distance (Gregory 2008). Objects interpreted to be at a far distance appear to be larger than those near to us. However, alternative accounts for several of these illusions have also been proposed instead positing that our perception of these illusions reflects the statistical properties of the visual environment (Howe and Purves 2004, 2005). The Shepard Tables (Figure 2F) influence our judgement of object size by exploiting inherent assumptions about perspective. Finally, some illusions like the rotating snakes (http://www.ritsumei.ac.jp/ akitaoka/index-e.html) motion that is not physically present in the image. Similarly, in the percept of illusory contours and amodal completion in images like the aforementioned Kanizsa figures, or the extrapolation of edges from abutting line segments (see the chapter by Kogo and van Ee, this volume, for an in-depth discussion of these processes), we perceive a faint luminance edge that can be of remarkable clarity simply due to the presence of inducing image components that imply the presence of a figure or an edge even though there is no physical luminance contrast. Thus, even very simple geometric stimulus features can influence and alter the contents of awareness, making us experience things that are not actually there. 
Naturally, this list is not exhaustive but meant to give an overview of the different types of visual illusions. One thing that they all share is that they affect the contents of our awareness by letting us see things that are at odds with physical reality. Many neuroimaging studies show that the neural representation of our perceived environment can be found even at relatively early stages of cortical visual processing. For example, activity produced by physically identical stimuli in primary visual cortex (V1) reflects their perceived size (Murray et al. 2006). Subsequent work shows that this was not solely due to larger responses to stimuli perceived as larger and that this effect required participants to attend to the stimulus (Fang, Boyaci, et al. 2008). More recently, this effect was further corroborated by the finding that the perceived size of a retinal afterimage is also reflected by V1 activity (Sperandio, Chouinard, et al. 2012). Intriguingly, the perceived size of afterimages is also susceptible to contextual size illusions (Sperandio, Lak, et al. 2012).

A

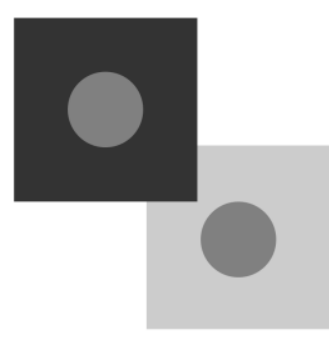

$\mathrm{D}$

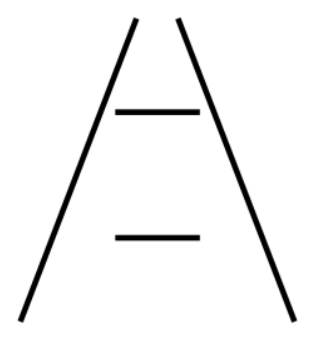

B

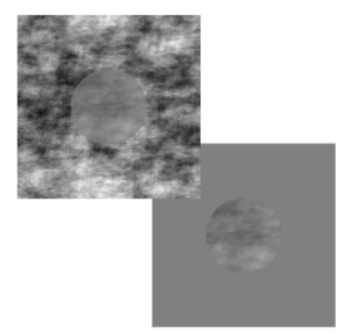

E

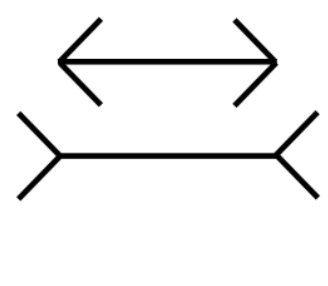

C

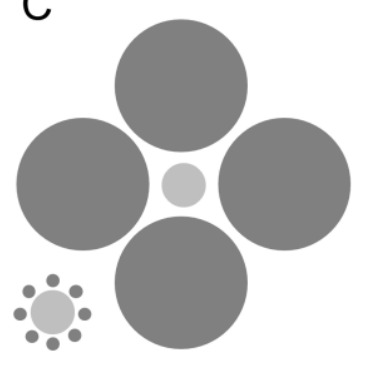

$\mathrm{F}$

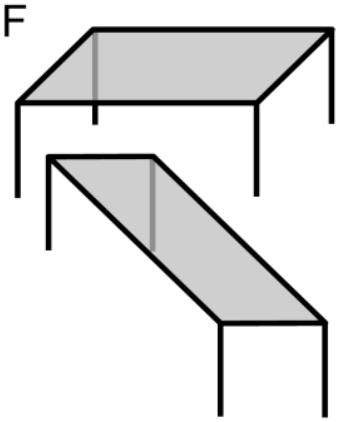

Figure 2. Visual illusions. A. Simultaneous brightness contrast. The luminance of the two circles is identical. B. Contrast suppression. The contrast in the two circular patches is identical. C. Ebbinghaus illusion. The size of the two light grey circles is the identical. D. Ponzo illusion. The length of the two horizontal lines is identical. E. Mueller-Lyer illusion. The length of horizontal section of the two arrows is identical. F. Shepard's Tables. The surface area of the two tables is identical.

Consistent with this, in our own experiments the Ebbinghaus illusion is reduced under dichoptic presentation when inducers and target stimuli are presented to different eyes (Song et al. 2011). Such absent or weak interocular transfer of an effect indicates that it must be at least partly mediated by early stages of visual processing where the information from the two eyes has not been fully combined, such as V1. We therefore hypothesised that the cortical surface area of V1, which varies quite considerably between individuals (Andrews et al. 1997; Dougherty et al. 2003), might covary with the strength of such size illusions. In particular, we reasoned that if the circuits mediating these illusions (lateral connections, feedback pathways) do not scale with V1 surface area, the strength of these illusions should thus be reduced in individuals with a larger V1. We measured the surface area of V1 in 30 individuals using functional MRI and retinotopic mapping procedures (Schwarzkopf et al. 2011) and compared that to the magnitude of the Ebbinghaus and a variant of the Ponzo illusion measured behaviourally in a psychophysics lab. As predicted, illusion magnitude was negatively correlated with V1 surface area. In subsequent experiments we further show that this correlation is present for both components of the Ebbinghaus stimulus, that is, both for contexts with both small and large inducers. Our results further support the 
interpretation that the cortical distance over which the contextual interaction occurs is a major factor determining illusion strength (Schwarzkopf and Rees 2013). While correlational studies like this cannot resolve the question of causality and the specific circuits mediating the illusion remain to be identified, our findings suggest that the surface area of $\mathrm{V} 1$ at least in part reflects the subjective awareness of object size.

All of the examples in this section thus far have been in the visual domain. As with perceptual science in general, vision has received most attention. However, there are also perceptual illusions in other sensory domains and it is important not to neglect these as of course all sensory input contributes to our subjective experience of the world. One example is the Aristotle illusion from the somatosensory modality that can occur when we cross our fingers (as when wishing somebody luck, or hoping for our Nature manuscript to be accepted for publication) and then touching a single marble so that it is held in between the two fingertips. One then has the experience (especially when moving the marble along the surface of a table or the floor) that there are two marbles, each touching one finger (Aristotle 1924). This percept may arise because in our interpretation of the somatosensory input the fingers are not normally crossed and so under typical conditions the sensation caused by this finger configuration would truly reflect the presence of two independent objects. Different sensory modalities may also interact to produce perceptual illusions, such as in the flash-beep illusion where the presence of a two sounds presented in brief succession simultaneously with a single visual flash can produce the percept of two independent flashes (Shams et al. 2000; Watkins et al. 2007). Interestingly, how prone an individual is to this illusion correlates with grey matter volume in early visual cortex (de Haas et al. 2012). Another example is the McGurk effect (McGurk and MacDonald 1976) that occurs when an auditory vocalisation of a syllable is presented together with an incongruent movie of a face vocalising a different syllable. The actual percept tends to be a mixture of the two modalities. Interestingly in the context of the topics discussed earlier, congruency between the visual face stimulus and the auditory vocalisation helps the face break through interocular suppression (Alsius and Munhall 2013); however, face stimuli rendered invisible through CFS did not produce the McGurk illusion suggesting that in order for a stimulus to exert multimodal effects it must be consciously perceived (Palmer and Ramsey 2012).

\section{Conclusion}

In this chapter, we outlined some of the ways in which consciousness interacts with the perceptual organisation of our sensory input. Not only does the brain's interpretation of stimuli influence whether or not they reach the focus of our awareness but we can also regard the way a scene is perceived to be a reflection of our subjective experience, the contents of awareness. We described a number of experiments investigating the processes by which our percepts are shaped by the brain and how to separate those functions that operate in the absence of awareness from those that require conscious processing. What kinds of sensory information can be interpreted without awareness remains unclear. The literature on this question is patchy with several studies investigating small aspects of unconscious perceptual processing, but a general theory tying together these findings is elusive.

It also still remains unresolved how different means of removing a stimulus from conscious access relate in terms of their neural mechanism and as such in how far they can be compared. The best experimental manipulations to study consciousness are those that keep the stimulus constant and instead rely on subjective differences in awareness to dissociate objective physical properties from subjective experience. This makes bistable stimuli and contextual illusions popular targets for experimental investigations, but this approach is not suited to address all questions. Therefore a more comprehensive comparison of different masking techniques will be instrumental in advancing our understanding of the role consciousness plays in perceptual organisation. 


\section{References}

Alais D, Blake R. 1999. Grouping visual features during binocular rivalry. Vision Res. 39:4341-4353.

Almeida J, Pajtas PE, Mahon BZ, Nakayama K, Caramazza A. 2013. Affect of the unconscious: visually suppressed angry faces modulate our decisions. Cogn Affect Behav Neurosci. 13:94-101.

Alsius A, Munhall KG. 2013. Detection of audiovisual speech correspondences without visual awareness. Psychol Sci. 24:423-431.

Andrews TJ, Halpern SD, Purves D. 1997. Correlated size variations in human visual cortex, lateral geniculate nucleus, and optic tract. J Neurosci. 17:2859-2868.

Anstis S, Kim J. 2011. Local versus global perception of ambiguous motion displays. J Vis. 11:13.

Aristotle. 1924. Metaphysics. Oxford, UK: Oxford University Press.

Aznar Casanova JA, Amador Campos JA, Moreno Sánchez M, Supér H. 2013. Onset time of binocular rivalry and duration of inter-dominance periods as psychophysical markers of ADHD. Perception. 42:16-27.

Bahrami B, Carmel D, Walsh V, Rees G, Lavie N. 2008a. Spatial attention can modulate unconscious orientation processing. Perception. 37:1520-1528.

Bahrami B, Carmel D, Walsh V, Rees G, Lavie N. 2008b. Unconscious orientation processing depends on perceptual load. J Vis. 8:12 1-10.

Baker DH, Graf EW. 2009. Natural images dominate in binocular rivalry. Proc Natl Acad Sci USA. 106:54365441.

Beck DM, Muggleton N, Walsh V, Lavie N. 2006. Right parietal cortex plays a critical role in change blindness. Cereb Cortex. 16:712-717.

Beck DM, Rees G, Frith CD, Lavie N. 2001. Neural correlates of change detection and change blindness. Nat Neurosci. 4:645-650.

Bonneh YS, Cooperman A, Sagi D. 2001. Motion-induced blindness in normal observers. Nature. 411:798801.

Brancucci A, Tommasi L. 2011. "Binaural rivalry": dichotic listening as a tool for the investigation of the neural correlate of consciousness. Brain Cogn. 76:218-224.

Breitmeyer BG, Ogmen H. 2000. Recent models and findings in visual backward masking: a comparison, review, and update. Percept Psychophys. 62:1572-1595.

Carmel D, Walsh V, Lavie N, Rees G. 2010. Right parietal TMS shortens dominance durations in binocular rivalry. Curr Biol. 20:R799-800.

Clifford CWG, Harris JA. 2005. Contextual modulation outside of awareness. Curr Biol. 15:574-578.

Cole GG, Kentridge RW, Heywood CA, Cole GG. 2004. Visual salience in the change detection paradigm: the special role of object onset. J Exp Psychol Hum Percept Perform. 30:464-477.

Conci M, Böbel E, Matthias E, Keller I, Müller HJ, Finke K. 2009. Preattentive surface and contour grouping in Kanizsa figures: evidence from parietal extinction. Neuropsychologia. 47:726-732.

Costello P, Jiang Y, Baartman B, McGlennen K, He S. 2009. Semantic and subword priming during binocular suppression. Conscious Cogn. 18:375-382.

De Gelder B, Morris JS, Dolan RJ. 2005. Unconscious fear influences emotional awareness of faces and voices. Proc Natl Acad Sci USA. 102:18682-18687.

De Haas B, Kanai R, Jalkanen L, Rees G. 2012. Grey matter volume in early human visual cortex predicts proneness to the sound-induced flash illusion. Proc Biol Sci. 279:4955-4961.

de-Wit LH, Kubilius J, Wagemans J, Op de Beeck HP. 2012. Bistable Gestalts reduce activity in the whole of V1, not just the retinotopically predicted parts. J Vis. 12.

Dehaene S, Changeux J-P, Naccache L, Sackur J, Sergent C. 2006. Conscious, preconscious, and subliminal processing: a testable taxonomy. Trends Cogn Sci (Regul Ed). 10:204-211.

Donner TH, Sagi D, Bonneh YS, Heeger DJ. 2008. Opposite neural signatures of motion-induced blindness in human dorsal and ventral visual cortex. J Neurosci. 28:10298-10310. 
Dougherty RF, Koch VM, Brewer AA, Fischer B, Modersitzki J, Wandell BA. 2003. Visual field representations and locations of visual areas $\mathrm{V} 1 / 2 / 3$ in human visual cortex. J Vis. 3:586-598.

Draganski B, Gaser C, Busch V, Schuierer G, Bogdahn U, May A. 2004. Neuroplasticity: changes in grey matter induced by training. Nature. 427:311-312.

Dumoulin SO, Hess RF. 2006. Modulation of V1 activity by shape: image-statistics or shape-based perception? J Neurophysiol. 95:3654-3664.

Einhäuser W, Martin KAC, König P. 2004. Are switches in perception of the Necker cube related to eye position? Eur J Neurosci. 20:2811-2818.

Faivre N, Berthet V, Kouider S. 2012. Nonconscious influences from emotional faces: a comparison of visual crowding, masking, and continuous flash suppression. Front Psychol. 3:129.

Falconbridge M, Ware A, MacLeod DIA. 2010. Imperceptibly rapid contrast modulations processed in cortex: Evidence from psychophysics. J. Vis. 10.

Fang F, Boyaci H, Kersten D, Murray SO. 2008. Attention-dependent representation of a size illusion in human V1. Curr Biol. 18:1707-1712.

Fang F, Kersten D, Murray SO. 2008. Perceptual grouping and inverse fMRI activity patterns in human visual cortex. J Vis. 8:2.1-9.

Field DJ. 1987. Relations between the statistics of natural images and the response properties of cortical cells. J Opt Soc Am A. 4:2379-2394.

Geisler WS. 2008. Visual perception and the statistical properties of natural scenes. Annual review of psychology. 59:167-192.

Geisler WS, Perry JS, Super BJ, Gallogly DP. 2001. Edge co-occurrence in natural images predicts contour grouping performance. Vision Res. 41:711-724.

Gillam B, Nakayama K. 1999. Quantitative depth for a phantom surface can be based on cyclopean occlusion cues alone. Vision Res. 39:109-112.

Gregory RL. 2008. Emmert's Law and the moon illusion. Spat Vis. 21:407-420.

Häkkinen J, Nyman G. 2001. Phantom surface captures stereopsis. Vision Res. 41:187-199.

Harris JJ, Schwarzkopf DS, Song C, Bahrami B, Rees G. 2011. Contextual illusions reveal the limit of unconscious visual processing. Psychol Sci. 22:399-405.

Haynes J-D, Driver J, Rees G. 2005. Visibility reflects dynamic changes of effective connectivity between V1 and fusiform cortex. Neuron. 46:811-821.

Haynes J-D, Rees G. 2005. Predicting the orientation of invisible stimuli from activity in human primary visual cortex. Nat Neurosci. 8:686-691.

He D, Kersten D, Fang F. 2012. Opposite modulation of high- and low-level visual aftereffects by perceptual grouping. Curr Biol. 22:1040-1045.

Howe CQ, Purves D. 2004. Size contrast and assimilation explained by the statistics of natural scene geometry. J Cogn Neurosci. 16:90-102.

Howe CQ, Purves D. 2005. The Müller-Lyer illusion explained by the statistics of image-source relationships. Proc Natl Acad Sci USA. 102:1234-1239.

Hugrass L, Crewther D. 2012. Willpower and conscious percept: volitional switching in binocular rivalry. PLOS ONE. 7:e35963.

Hunt JJ, Mattingley JB, Goodhill GJ. 2012. Randomly oriented edge arrangements dominate naturalistic arrangements in binocular rivalry. Vision Res. 64:49-55.

James W. 1890. The principles of psychology. New York : Holt.

Jiang Y, Costello P, He S. 2007. Processing of invisible stimuli: advantage of upright faces and recognizable words in overcoming interocular suppression. Psychol Sci. 18:349-355.

Joo SJ, Boynton GM, Murray SO. 2012. Long-range, pattern-dependent contextual effects in early human visual cortex. Curr Biol. 22:781-786.

Kanai R, Bahrami B, Rees G. 2010. Human parietal cortex structure predicts individual differences in perceptual rivalry. Curr Biol. 20:1626-1630. 
Kanai R, Carmel D, Bahrami B, Rees G. 2011. Structural and functional fractionation of right superior parietal cortex in bistable perception. Curr Biol. 21:R106-107.

Kentridge RW, Heywood CA, Weiskrantz L. 1999. Attention without awareness in blindsight. Proc Biol Sci. 266:1805-1811.

Kinoshita M, Gilbert CD, Das A. 2009. Optical imaging of contextual interactions in v1 of the behaving monkey. J Neurophysiol. 102:1930-1944.

Koch C, Tsuchiya N. 2007. Attention and consciousness: two distinct brain processes. Trends Cogn Sci (Regul Ed). 11:16-22.

Kogo N, Strecha C, Van Gool L, Wagemans J. 2010. Surface construction by a 2-D differentiation-integration process: a neurocomputational model for perceived border ownership, depth, and lightness in Kanizsa figures. Psychol Rev. 117:406-439.

Kok P, Jehee JFM, de Lange FP. 2012. Less is more: expectation sharpens representations in the primary visual cortex. Neuron. 75:265-270.

Krug K, Brunskill E, Scarna A, Goodwin GM, Parker AJ. 2008. Perceptual switch rates with ambiguous structure-from-motion figures in bipolar disorder. Proc Biol Sci. 275:1839-1848.

Lamme VA, Roelfsema PR. 2000. The distinct modes of vision offered by feedforward and recurrent processing. Trends Neurosci. 23:571-579.

Lamme VAF. 2003. Why visual attention and awareness are different. Trends Cogn Sci (Regul Ed). 7:12-18.

Lamme VAF. 2006. Towards a true neural stance on consciousness. Trends Cogn Sci (Regul Ed). 10:494-501.

Leopold DA, Wilke M, Maier A, Logothetis NK. 2002. Stable perception of visually ambiguous patterns. Nat Neurosci. 5:605-609.

Lin Z, He S. 2009. Seeing the invisible: the scope and limits of unconscious processing in binocular rivalry. Prog Neurobiol. 87:195-211.

Ling S, Blake R. 2009. Suppression During Binocular Rivalry Broadens Orientation Tuning. Psychol Sci.

Liu L, Stevenson SB, Schor CM. 1994. Quantitative stereoscopic depth without binocular correspondence. Nature. 367:66-69.

Lumer ED, Friston KJ, Rees G. 1998. Neural correlates of perceptual rivalry in the human brain. Science. 280:1930-1934.

Macknik SL, Livingstone MS. 1998. Neuronal correlates of visibility and invisibility in the primate visual system. Nat Neurosci. 1:144-149.

Macknik SL, Martinez-Conde S. 2007. The role of feedback in visual masking and visual processing. Adv Cogn Psychol. 3:125-152.

Maehara G, Huang P-C, Hess RF. 2009. Importance of phase alignment for interocular suppression. Vision Res. 49:1838-1847.

Maruya K, Blake R. 2009. Spatial spread of interocular suppression is guided by stimulus configuration. Perception. 38:215-231.

Mattingley JB, Davis G, Driver J. 1997. Preattentive filling-in of visual surfaces in parietal extinction. Science. 275:671-674.

McGurk H, MacDonald J. 1976. Hearing lips and seeing voices. Nature. 264:746-748.

Meng M, Tong F. 2004. Can attention selectively bias bistable perception? Differences between binocular rivalry and ambiguous figures. $J$ Vis. 4:539-551.

Miller SM, Gynther BD, Heslop KR, Liu GB, Mitchell PB, Ngo TT, Pettigrew JD, Geffen LB. 2003. Slow binocular rivalry in bipolar disorder. Psychol Med. 33:683-692.

Miller SM, Hansell NK, Ngo TT, Liu GB, Pettigrew JD, Martin NG, Wright MJ. 2010. Genetic contribution to individual variation in binocular rivalry rate. Proc Natl Acad Sci USA. 107:2664-2668.

Motoyoshi I, Hayakawa S. 2010. Adaptation-induced blindness to sluggish stimuli. J Vis. 10:16.1-8.

Moutoussis K, Zeki S. 2002. The relationship between cortical activation and perception investigated with invisible stimuli. Proc Natl Acad Sci USA. 99:9527-9532. 
Mudrik L, Breska A, Lamy D, Deouell LY. 2011. Integration without awareness: expanding the limits of unconscious processing. Psychol Sci. 22:764-770.

Murray SO, Boyaci H, Kersten D. 2006. The representation of perceived angular size in human primary visual cortex. Nat Neurosci. 9:429-434.

Murray SO, Kersten D, Olshausen BA, Schrater P, Woods DL. 2002. Shape perception reduces activity in human primary visual cortex. Proc Natl Acad Sci USA. 99:15164-15169.

Nagamine M, Yoshino A, Miyazaki M, Takahashi Y, Nomura S. 2009. Difference in binocular rivalry rate between patients with bipolar I and bipolar II disorders. Bipolar Disord. 11:539-546.

O'Craven KM, Downing PE, Kanwisher N. 1999. fMRI evidence for objects as the units of attentional selection. Nature. 401:584-587.

Ooi TL, He ZJ. 1999. Binocular rivalry and visual awareness: the role of attention. Perception. 28:551-574.

Palmer TD, Ramsey AK. 2012. The function of consciousness in multisensory integration. Cognition. 125:353-364.

Pettigrew JD, Miller SM. 1998. A "sticky" interhemispheric switch in bipolar disorder? Proc Biol Sci. 265:2141-2148.

Ramachandran VS, Gregory RL. 1991. Perceptual filling in of artificially induced scotomas in human vision. Nature. 350:699-702.

Rao RP, Ballard DH. 1999. Predictive coding in the visual cortex: a functional interpretation of some extraclassical receptive-field effects. Nat Neurosci. 2:79-87.

Rees G, Kreiman G, Koch C. 2002. Neural correlates of consciousness in humans. Nat Rev Neurosci. 3:261270.

Roberts B, Harris MG, Yates TA. 2005. The roles of inducer size and distance in the Ebbinghaus illusion (Titchener circles). Perception. 34:847-856.

Roelfsema PR, Lamme VA, Spekreijse H. 1998. Object-based attention in the primary visual cortex of the macaque monkey. Nature. 395:376-381.

Said CP, Egan RD, Minshew NJ, Behrmann M, Heeger DJ. 2013. Normal binocular rivalry in autism: implications for the excitation/inhibition imbalance hypothesis. Vision Res. 77:59-66.

Schölvinck ML, Rees G. 2009. Attentional influences on the dynamics of motion-induced blindness. J.Vis. 9:1-9.

Schölvinck ML, Rees G. 2010. Neural correlates of motion-induced blindness in the human brain. J Cogn Neurosci. 22:1235-1243.

Schurger A, Pereira F, Treisman A, Cohen JD. 2010. Reproducibility distinguishes conscious from nonconscious neural representations. Science. 327:97-99.

Schwarzkopf DS, Rees G. 2010. Interpreting local visual features as a global shape requires awareness. Proc Biol Sci.

Schwarzkopf DS, Rees G. 2013. Subjective size perception depends on central visual cortical magnification in human v1. PLoS ONE. 8:e60550.

Schwarzkopf DS, Silvanto J, Gilaie-Dotan S, Rees G. 2010. Investigating object representations during change detection in human extrastriate cortex. Eur J Neurosci. 32:1780-1787.

Schwarzkopf DS, Song C, Rees G. 2011. The surface area of human V1 predicts the subjective experience of object size. Nat Neurosci. 14:28-30.

Shams L, Kamitani Y, Shimojo S. 2000. Illusions. What you see is what you hear. Nature. 408:788.

Shannon RW, Patrick CJ, Jiang Y, Bernat E, He S. 2011. Genes contribute to the switching dynamics of bistable perception. J Vis. 11.

Silverman ME, Mack A. 2006. Change blindness and priming: When it does and does not occur. Conscious Cogn. 15:409-422.

Simoncelli EP, Olshausen BA. 2001. Natural image statistics and neural representation. Annual review of neuroscience. 24:1193-1216. 
Sobel KV, Blake R. 2003. Subjective contours and binocular rivalry suppression. Vision Res. 43:1533-1540.

Song C, Schwarzkopf DS, Rees G. 2011. Interocular induction of illusory size perception. BMC Neurosci. 12:27.

Sperandio I, Chouinard PA, Goodale MA. 2012. Retinotopic activity in V1 reflects the perceived and not the retinal size of an afterimage. Nat Neurosci. 15:540-542.

Sperandio I, Lak A, Goodale MA. 2012. Afterimage size is modulated by size-contrast illusions. J Vis. 12.

Stanley DA, Rubin N. 2003. fMRI activation in response to illusory contours and salient regions in the human lateral occipital complex. Neuron. 37:323-331.

Sterzer P, Haynes J-D, Rees G. 2008. Fine-scale activity patterns in high-level visual areas encode the category of invisible objects. J Vis. 8:10.1-12.

Stewart LH, Ajina S, Getov S, Bahrami B, Todorov A, Rees G. 2012. Unconscious evaluation of faces on social dimensions. J Exp Psychol Gen. 141:715-727.

Tse PU, Martinez-Conde S, Schlegel AA, Macknik SL. 2005. Visibility, visual awareness, and visual masking of simple unattended targets are confined to areas in the occipital cortex beyond human V1/V2. Proc Natl Acad Sci USA. 102:17178-17183.

Tsuchiya N, Koch C. 2005. Continuous flash suppression reduces negative afterimages. Nat Neurosci. 8:1096-1101.

Turatto M, Sandrini M, Miniussi C. 2004. The role of the right dorsolateral prefrontal cortex in visual change awareness. Neuroreport. 15:2549-2552.

Van Ee R, van Boxtel JJA, Parker AL, Alais D. 2009. Multisensory congruency as a mechanism for attentional control over perceptual selection. J Neurosci. 29:11641-11649.

Van Loon AM, Knapen T, Scholte HS, St John-Saaltink E, Donner TH, Lamme VAF. 2013. GABA Shapes the Dynamics of Bistable Perception. Curr Biol. 23:823-827.

Vuilleumier P, Armony JL, Driver J, Dolan RJ. 2003. Distinct spatial frequency sensitivities for processing faces and emotional expressions. Nat Neurosci. 6:624-631.

Wandell BA, Dumoulin SO, Brewer AA. 2007. Visual field maps in human cortex. Neuron. 56:366-383.

Wang L, Weng X, He S. 2012. Perceptual grouping without awareness: superiority of Kanizsa triangle in breaking interocular suppression. PLOS ONE. 7:e40106.

Wannig A, Stanisor L, Roelfsema PR. 2011. Automatic spread of attentional response modulation along Gestalt criteria in primary visual cortex. Nat Neurosci. 14:1243-1244.

Watkins S, Shams L, Josephs O, Rees G. 2007. Activity in human V1 follows multisensory perception. Neuroimage. 37:572-578.

Weil RS, Kilner JM, Haynes JD, Rees G. 2007. Neural correlates of perceptual filling-in of an artificial scotoma in humans. Proc Natl Acad Sci USA. 104:5211-5216.

Weil RS, Watkins S, Rees G. 2008. Neural correlates of perceptual completion of an artificial scotoma in human visual cortex measured using functional MRI. Neuroimage. 42:1519-1528.

Williams MA, Morris AP, McGlone F, Abbott DF, Mattingley JB. 2004. Amygdala responses to fearful and happy facial expressions under conditions of binocular suppression. J Neurosci. 24:2898-2904.

Winston JS, Vuilleumier P, Dolan RJ. 2003. Effects of low-spatial frequency components of fearful faces on fusiform cortex activity. Curr Biol. 13:1824-1829.

Wismeijer DA, Erkelens CJ, van Ee R, Wexler M. 2010. Depth cue combination in spontaneous eye movements. J Vis. 10:25.

Wismeijer DA, van Ee R, Erkelens CJ. 2008. Depth cues, rather than perceived depth, govern vergence. Exp Brain Res. 184:61-70.

Wokke ME, Vandenbroucke ARE, Scholte HS, Lamme VAF. 2013. Confuse your illusion: feedback to early visual cortex contributes to perceptual completion. Psychol Sci. 24:63-71.

Wolfe JM. 1984. Reversing ocular dominance and suppression in a single flash. Vision Res. 24:471-478.

Yang E, Blake R. 2012. Deconstructing continuous flash suppression. J Vis. 12:8. 
Yang E, Zald DH, Blake R. 2007. Fearful expressions gain preferential access to awareness during continuous flash suppression. Emotion. 7:882-886.

Yeh Y-Y, Yang C-T. 2009. Is a pre-change object representation weakened under correct detection of a change? Conscious Cogn. 18:91-102.

Zaretskaya N, Anstis S, Bartels A. 2013. Parietal cortex mediates conscious perception of illusory gestalt. J Neurosci. 33:523-531.

Zaretskaya N, Thielscher A, Logothetis NK, Bartels A. 2010. Disrupting parietal function prolongs dominance durations in binocular rivalry. Curr Biol. 20:2106-2111.

Zhaoping L. 2008. Attention capture by eye of origin singletons even without awareness--a hallmark of a bottom-up saliency map in the primary visual cortex. $J$ Vis. 8:1.1-18.

Zhou W, Chen D. 2009. Binaral rivalry between the nostrils and in the cortex. Curr Biol. 19:1561-1565.

Zimba LD, Blake R. 1983. Binocular rivalry and semantic processing: out of sight, out of mind. J Exp Psychol Hum Percept Perform. 9:807-815. 